\title{
FC-TLBO: fully constrained meta-heuristic algorithm for abundance estimation using linear mixing model
}

\author{
OMPRAKASH TEMBHURNE* and DEEPTI SHRIMANKAR \\ Department of Computer Science and Engineering, Visvesvaraya National Institute of Technology, \\ Nagpur 440010, India \\ e-mail: owtembhurne@gmail.com
}

MS received 21 June 2016; revised 16 November 2016; accepted 17 January 2017

\begin{abstract}
A study of abundance estimation has vital importance in spectral unmixing of hyperspectral image. Recently, various methods have been proposed for spectral unmixing to achieve higher performance using an evolutionary approach. However, these methods are based on unconstrained optimisation problems. Their performance was also based on proper tuning parameters. We have proposed a new non-parametric algorithm using teaching-learning-based optimisation technique with an inbuilt constraints maintenance mechanism using the linear mixing model. In this approach, the unmixing problem is transformed into a combinatorial optimisation problem by introducing abundance sum to one constraint and abundance non-negative constraint. A comparative analysis of the proposed algorithm is conducted with other two state-of-the-art algorithms. Experimental results in known and unknown environments with varying signal-to-noise ratio on simulated and real hyperspectral data demonstrate that the proposed method outperforms the other methods.
\end{abstract}

Keywords. Abundance non-negative constraint (ANC); abundance sum to one constraint (ASC); hyperspectral unmixing; meta-heuristic approach; teaching-learning-based optimisation (TLBO).

\section{Introduction}

Hyperspectral image [1] is a set of images concerned with capturing reflectances across the electromagnetic spectrum. These bands typically span visible and near-infrared spectrum, although some sensors have bands in short-wavelength infrared ranges, mid-wavelength infrared ranges and thermal infrared ranges. The hyperspectral image-capturing instruments such as airborne visible infrared imaging spectrometer (AVIRIS) can record the visible and nearinfrared spectrum $(0.4-2.5 \mu \mathrm{m})$ of area $2-12 \mathrm{~km}$ wide and several kilometres long using 224 spectral bands of the continuous interval [2]. Many application uses hyperspectral scenes for mineral identification, vegetation analysis, geographical survey, disaster management and identification of a target in military applications. The analysis and interpretation of hyperspectral scenes have become a viable area of research due to its real-time applications.

Satellite hyperspectral images contain data of large geographic areas. Due to the low spatial resolution of sensors and asymmetrical geometric conditions (e.g., uneven earth surface, presence of moisture in air and cloudy conditions), pixels in images contain a mixture of more than two endmembers. In hyperspectral scenes, multiple interactions among scattering photons are introduced

*For correspondence to describe mixing processes. The radioactive transfer (RT) theory contributes in the calculation of mixing effect. However, mathematical calculations of mixing effect using this theory are a tedious task for a real-time application [3].

Many researchers have attempted to solve the problem of mathematical tractability of RT theory using the principle of approximate analysis. The approximate analysis is formulated using various mixing models that are explained in detail in [4]. In [5] Dobigeon et al explains the mixing effect on reflectance due to various geometric conditions. Use of mixing models is dependent on the application, for example, if features captured by a hyperspectral image are macroscopic and linearly separable, linear mixing model (LMM) creates remarkable results. Similarly, when the features are microscopic, multi-layered, intimate mixture and linearly non-separable, bi-linear mixing model (BMM) and polynomial post-nonlinear mixing model (PPNM) create remarkable results. However, due to the strong physical basis and mathematical simplicity, LMM is widely utilized by most of the researchers $[5,6]$.

Spectral unmixing has vital importance in hyperspectral data analysis and applications. It involves separation of the reflected spectrum into endmember spectra and their relative abundance fractions. Endmembers are considered pure pixels present in the scene and abundance fractions are their proportions due to mixing effect. In LMM, the spectrum of mixed pixels in hyperspectral scene is assumed to be the 
linear combination of $K$ deterministic endmembers $\left(E_{j}\right)$ with fractional abundance $s_{i, j}$ such that

$$
\begin{aligned}
& f(E, s)=\sum_{j=1}^{K} s_{i j} E_{j}+n \quad i=1,2, \ldots, M \\
& \text { s.t., (ANC), } s_{i, j} \geq 0 \text { and (ASC), } \sum_{j=1}^{K} s_{j}=1
\end{aligned}
$$

where random vector $\mathrm{n}$ represents the addition of Gaussian noise and $M$ is the total number of pixels present in a hyperspectral image [7].

Once the endmembers are known in advance either by endmember extraction algorithms (EEA) or by the available online libraries (figure 1), abundance fractions can be commonly obtained by fully constrained least squares method (FCLS) [7]. However, it is notable that the result of FCLS strongly depends on the accuracy of the known endmembers involved in hyperspectral unmixing [8]. Markov chain Monte Carlo (MCMC) algorithm also contributed to the theory of spectral unmixing [9]. However, it shows a remarkable variation in abundance estimated for the same input in different runs.

Despite much advancement in the field of hyperspectral unmixing, automated evaluation of abundance fractions from densely populated hyperspectral images by maintaining abundance sum to one constraint (ASC), abundance non-negative constraint (ANC) is challenging. Several successful statistical, geometric, heuristic and regressionbased models have been proposed in the recent past. Particle swarm optimisation is used to optimise the value of the objective function for spectral unmixing [10]. However, it was defined for unconstrained optimisation, and its performance depends on choosing proper values of inertia weight and acceleration constant for updating particle velocity in the search space. In refs. [11-16], several evolutionary algorithms (e.g., ant colony optimisation (ACO) and differential evolution (DE)) based on the random generation of the population for searching solution in problem space are used for hyperspectral image analysis. The solution of problems using these algorithms also requires proper adjustable parameters. Therefore, the fully

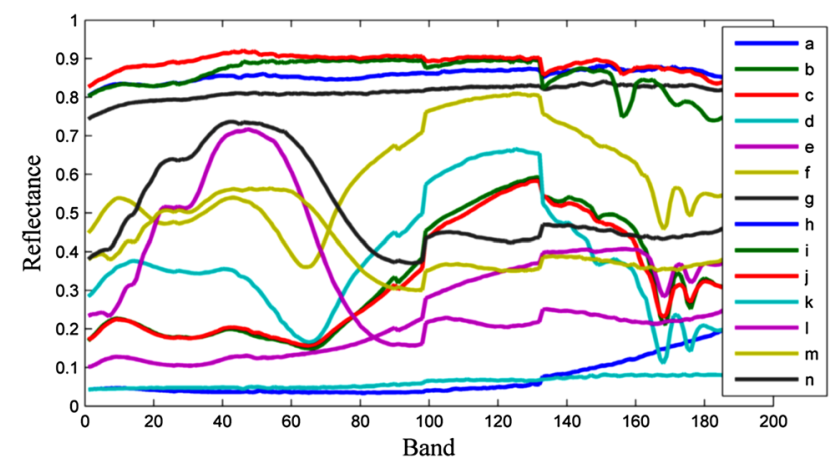

Figure 1. Spectral signature of endmember randomly selected from United States geological Survey (USGS) spectral library [20]. constrained non-parametric evolutionary approach for abundance estimation is always a challenging problem.

Since randomness is a machine-specific parameter, it is very difficult to maintain the ASC and ANC in abundance estimation using evolutionary approach. In [17], a new approach based on penalty function to maintain ASC and ANC constraints on model-based approach is proposed. However, it has several other drawbacks like; convergence of solution depends on the initial parameters, lack of feedback mechanism, and intense mathematical calculation.

In this paper, a new approach for constrained hyperspectral unmixing using teaching-learning-based optimisation technique (TLBO) [18] is proposed. It is a nonparametric approach and also depends on the feedback mechanism for searching the best solution in solution space. The abundance fractions are the unknown parameters. Machine-specific function randomly generates the population of unknown parameters. The random population generation approaches a state that the proposed approach is independent of initial parameters. The ASC and ANC are managed by (i) restricting machine-specific function to generate random population in the limit of physical constraints and (ii) restricting updated unknown parameters in limits of physical constraints using clipping operations. The experimental results in a known and unknown environment show that the proposed algorithm achieves not only better unmixing accuracy but also robust to noise.

Subsequently, section 2 describes the methods and materials used for the proposed approach. Section 3 describes the detailed methodology of proposed FC-TLBO. Section 4 contains the experimental results on simulated and real hyperspectral images. While section 5 provides the conclusion and prospective work.

\section{Methods and materials}

\subsection{Teaching-learning based optimisation (TLBO)}

TLBO is a meta-heuristic approach inspired by classroom teaching-learning process [18]. The objective function of TLBO is to improve the overall result of the class. It is a population-based optimisation technique which considers the random population of solutions to proceed for a globally acceptable solution. The population is the total number of random solutions generated in the solution space. The group of learners is considered population. TLBO is based on two phases of learning: 'teacher phase' and 'learner phase'. The most knowledgeable person among learners is considered teacher. In 'teacher phase', the teacher improves the knowledge of learners. In 'learner phase', learners improve their knowledge by interacting among themselves. The improvement in knowledge of learners is a continuous process. The feedback mechanism takes care of assurance of improvement while updating the knowledge of learners. 
The process continues until the group solutions reach to the global optimum solution.

\section{Proposed algorithm}

TLBO inspires FC-TLBO. In this problem, design variables are abundance fractions and the objective function is the minimisation problem described in Eq. (2), where $y$ is the true pixel reflectance and $f(E, s)$ is the estimated pixel reflectance according to the parameters described in Eq. (1).

$$
\operatorname{fun}(y, E, s)=\arg \min _{\mathrm{s}}\left\{\frac{1}{2}\|y-f(E, s)\|^{2}\right\}
$$

The proposed FC-TLBO segregates into three parts: 'initialisation phase', 'teacher phase' and 'learner phase'.

\subsection{Initialisation phase}

The initialisation phase consists of two parts (i) initialisation of parameters required for convergence and maximum size of population, (ii) initialisation of population of abundance fractions using dirichlet_rnd function [19] that maintain ASC and ANC. dirichlet_rnd function ${ }^{1}$ is defined by José Nascimento and José Bioucas-Dias. $\mathrm{R}=$ dirichlet_rnd $(A, K)$ returns random matrix $R$ from Dirichlet distribution. The size of $R$ is $(N \times K)$, where $N$ is the size of the population and $K$ is the total number of endmembers.

\subsection{Teacher phase}

The abundance fractions are actual parameters involved in the objective function of the given optimisation problem. The best value of the objective function is the best solution. The parameter that generates the best solution in the entire population is considered as a teacher and is well described by Eq. (3). The initial first value of abundance among the randomly generated population of $N$ abundances is considered the teacher's abundance. The value of an objective function using remaining abundances from the entire population compares with the value of an objective function using teacher abundance. The abundance that creates the smallest value of the objective function from the entire population is considered as the teacher's abundance.

$$
s_{i, j}^{T}= \begin{cases}s_{1, j} & \text { if }\left(\operatorname{fun}\left(y, E, s_{1, j}\right)<\operatorname{fun}\left(y, E, s_{i, j}\right)\right) \\ s_{i, j} & \text { otherwise }\end{cases}
$$

During this phase, the teacher tries to improve learners to create the best value of an objective function using their individual capability. The following four steps govern the updating rules of the individual learner.
Step 1. Calculate the mean value of abundance of entire population using Eq. (4).

$$
\mu_{1, j}=\frac{\sum_{i=1}^{N} s_{i, j}}{N},\{\forall j=1 \ldots K
$$

Step 2. Randomly select teaching factor using Eq. (5). The value of teaching factor is either one or two, depending on machine-dependent $\operatorname{rand}(0,1)$ function [18]. The value of $\operatorname{rand}(0,1)$ function is in between 0 and 1 .

$$
\mathrm{TF}=\operatorname{round}(1+\operatorname{rand}(0,1))
$$

Step 3. Create new values for learners of entire population using Eq. (6). The new value of abundance fraction depends on knowledge of teacher abundance, teaching factor, mean abundance and its original value. The $\operatorname{rand}([K, 1])$ function is used to create arrays of $K$ random values between 0 and 1 . ANC and ASC are managed using clipping operations described in Eqs. (7) and (8). If the summation of fractional values of new abundance is greater than one, then round it off to one. If it is less than zero, then round it off to zero.

$$
\begin{gathered}
s_{i, j}^{\text {new }}=s_{i, j}+\operatorname{rand}([K, 1]) \times s_{i, j}^{T}-\mathrm{TF} \times \mu_{1, j} \\
s_{i, j}^{\text {new }}=\max \left(\sum_{j=1}^{K} s_{i, j}^{\text {new }}, 0\right) \\
s_{i, j}^{\text {new }}=\min \left(\sum_{j=1}^{K} s_{i, j}^{\text {new }}, 1\right)
\end{gathered}
$$

Step 4. Update the learners of entire population if new values are better than previous ones using Eq. (9). This step uses a feedback mechanism for updating the original values of abundances.

$$
\left.\begin{array}{l}
\text { if }\left(\operatorname{fun}\left(y, E, s_{i, j}^{\text {new }}\right)<\operatorname{fun}\left(y, E, s_{i, j}\right)\right) \\
\text { Then } s_{i, j}=s_{i, j}^{\text {new }} \\
\text { else } s_{i, j}=s_{i, j}
\end{array}\right\}
$$

\subsection{Learner phase}

Learners interact randomly among themselves to improve their capability to minimise the value of the objective function. The following three steps govern the interaction phenomenon of learners.

Step 1. Randomly select a learner (rand) from the entire population. A learner can be any abundance from a population of randomly generated and updated abundances.

Step 2. Create new values of learners for entire populations. If the value of an objective function using learner (rand) is less than the value of the objective function of other learners then create its new value according to Eq. (10). To manage ASC and ANC use clipping operations in Eqs. (7) and (8). 


$$
\left.\begin{array}{l}
\text { if }\left(\operatorname{fun}\left(y, E, s_{i, j}^{\mathrm{rand}}\right)<\operatorname{fun}\left(y, E, s_{i, j}\right)\right) \\
\text { Then } s_{i, j}^{\text {new }}=s_{i, j}+\operatorname{rand}([K, 1]) \times\left(s_{i, j}^{\text {rand }}-s_{i, j}\right) \\
\text { else } s_{i, j}^{\text {new }}=s_{i, j}+\operatorname{rand}([K, 1]) \times\left(s_{i, j}-s_{i, j}^{\text {rand }}\right)
\end{array}\right\}
$$

Step 3. Update the value of learners if the new values are better than the previous one using Eq. (11).

$$
\left.\begin{array}{l}
\text { if }\left(\operatorname{fun}\left(y, E, s_{i, j}^{\text {new }}\right)<\operatorname{fun}\left(y, E, s_{i, j}\right)\right) \\
\text { Then } s_{i, j}=s_{i, j}^{\text {new }} \\
\text { else } s_{i, j}=s_{i, j}
\end{array}\right\}
$$

Being an iterative process, the convergence of FC-TLBO is also a concern. Along with increasing iterations, the error in abundance estimation (RMSE) and reconstruction error (RE) both were gradually reduced until they approach a stable state. If the difference of log-likelihood values of the objective function between two successive iterations is less than 0.00001 for 100 attempts, we consider it as a convergence situation. Figure 2 gives the flowchart of FCTLBO.

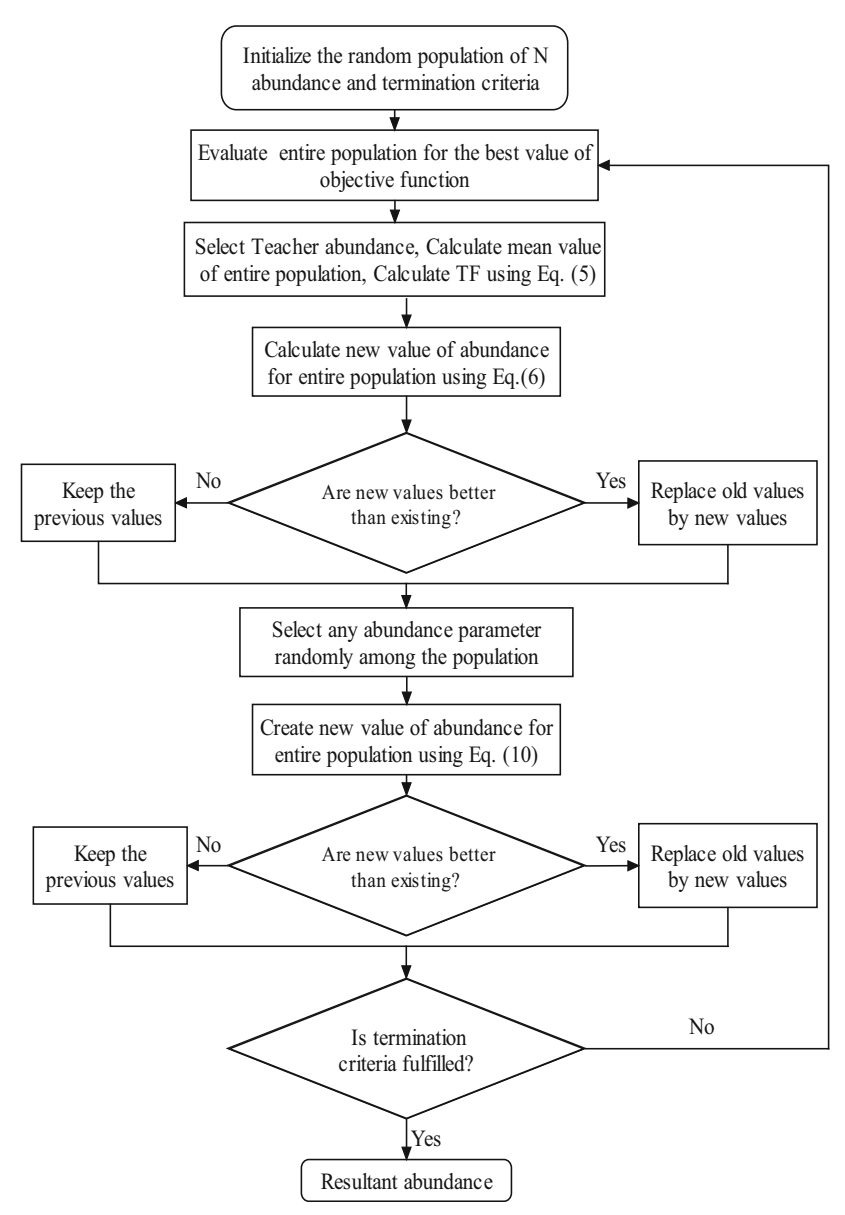

Figure 2. Flowchart of FC-TLBO algorithm.

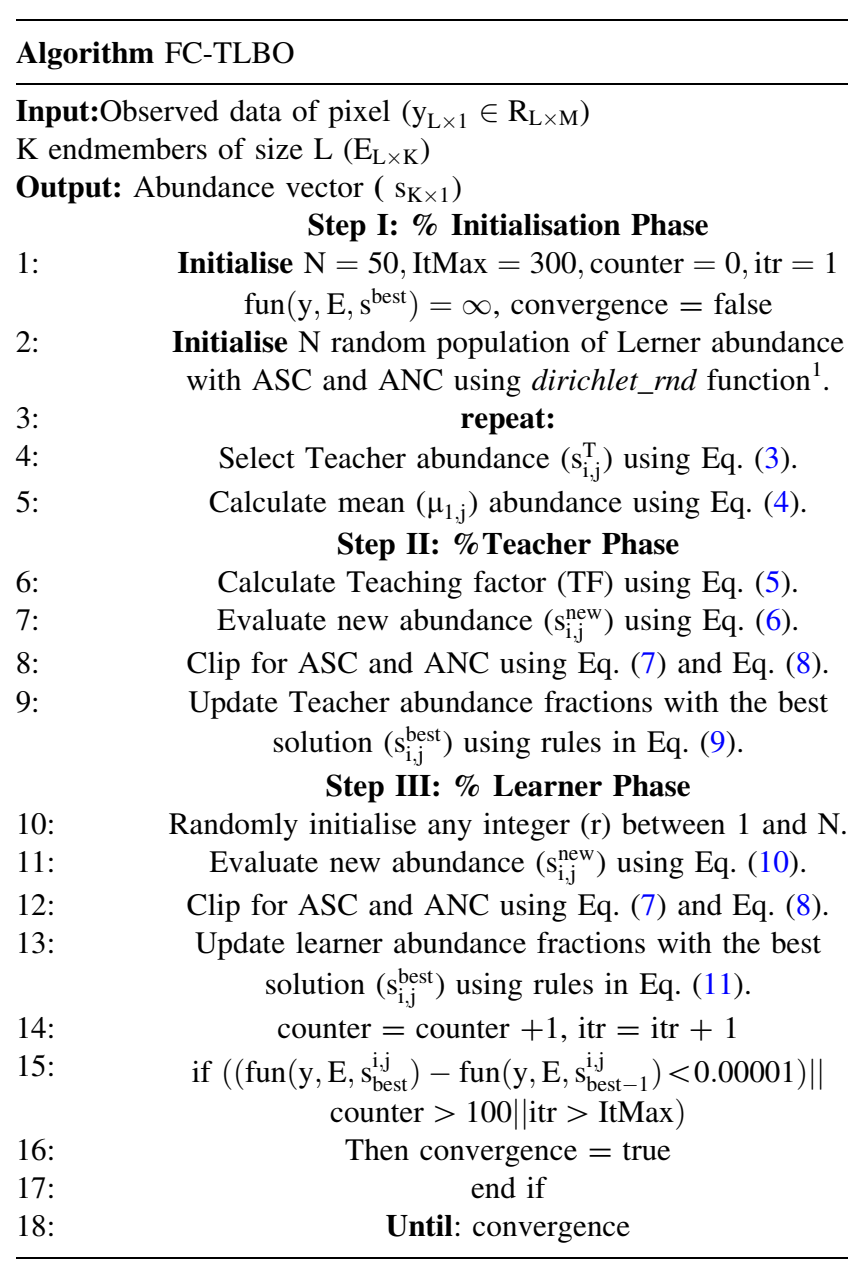

\section{Experiments and analysis}

The experiments are conducted on two different types of hyperspectral images, that is, simulated scenes and widely used real hyperspectral images such as Cuprite dataset, University of Pavia dataset and Indian Pines dataset.

Two metrics, including the root-mean-square error (RMSE) and reconstruction error (RE), are adapted to measure the performance of unmixing. RMSE between the true $\left(s_{i}\right)$ and estimated fractional abundance $\left(\tilde{s}_{i}\right)$ of the simulated scene is mathematically characterised as

$$
\mathrm{RMSE}=\sqrt{\frac{1}{M}\left(\sum_{i=1}^{M}\left\|s_{i}-\tilde{s}_{i}\right\|^{2}\right)}
$$

Reconstruction Error (RE) between the real $\left(y_{i}\right)$ and simulated scene $\left(\tilde{y}_{i}\right)$ is defined by

$$
\mathrm{RE}=\sqrt{\frac{1}{M}\left(\sum_{i=1}^{M}\left\|y_{i}-\tilde{y}_{i}\right\|^{2}\right)}
$$



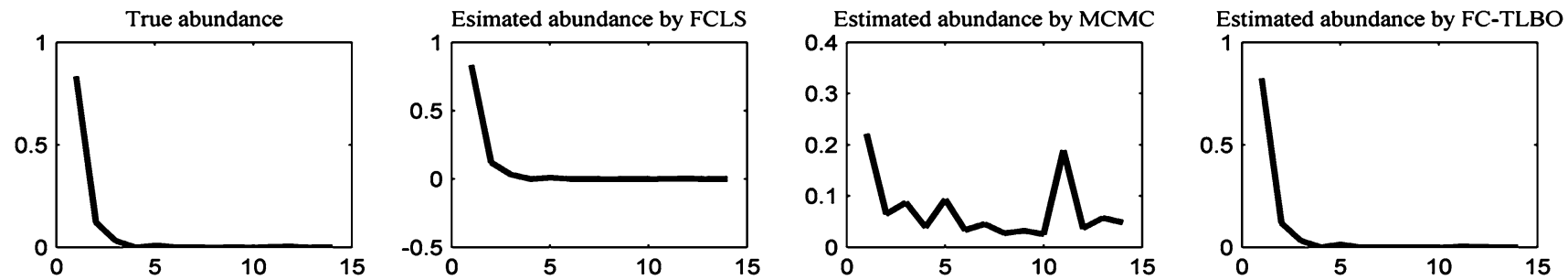

Figure 3. Comparison of true abundance fractions and estimated abundance fraction by FCLS, MCMC and FC-TLBO for randomly selected pixel from simulated scene when the number of endmembers is equal to 14 .
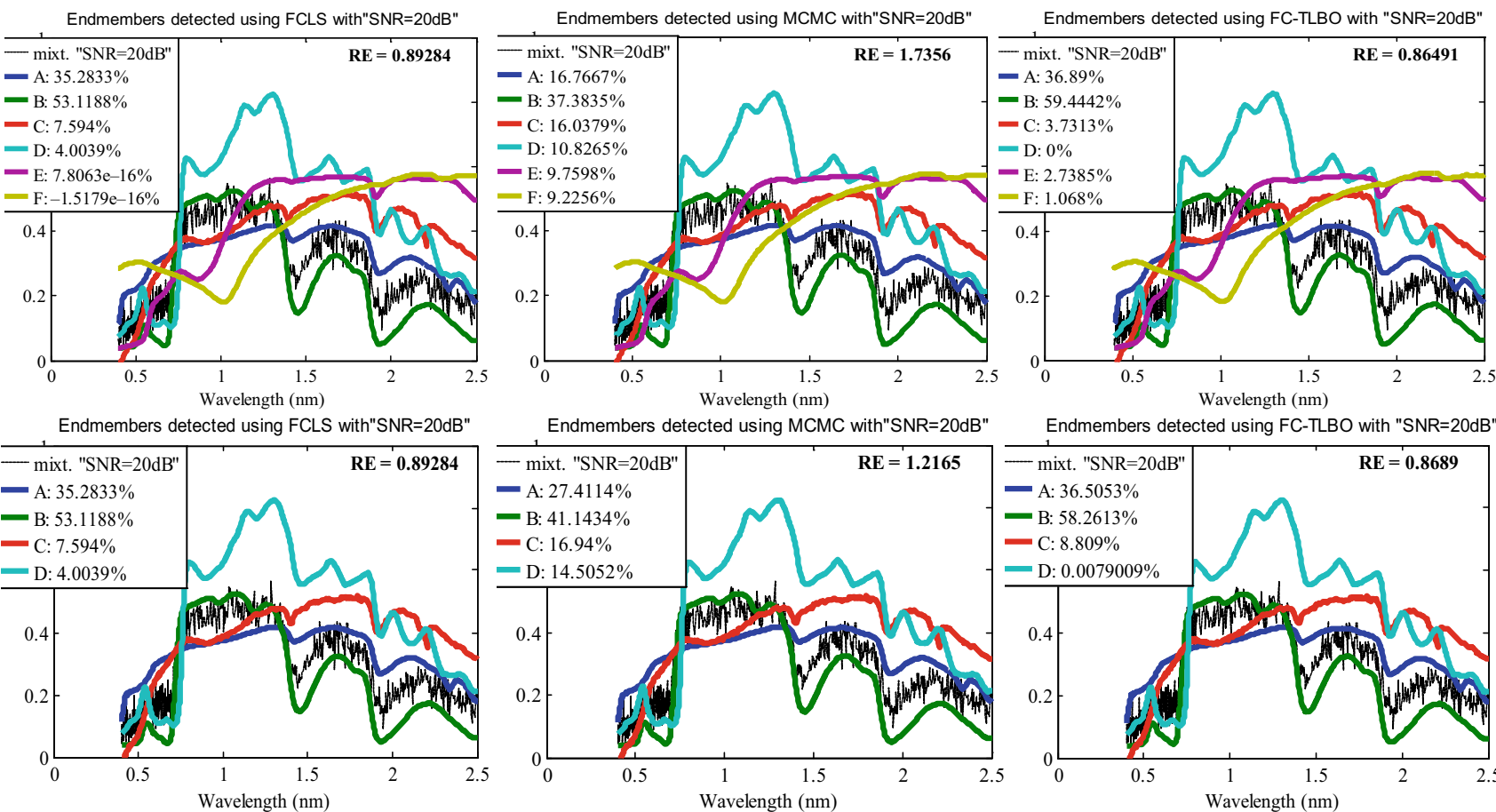

Endmembers detected using FC-TLBO with "SNR=20dB"
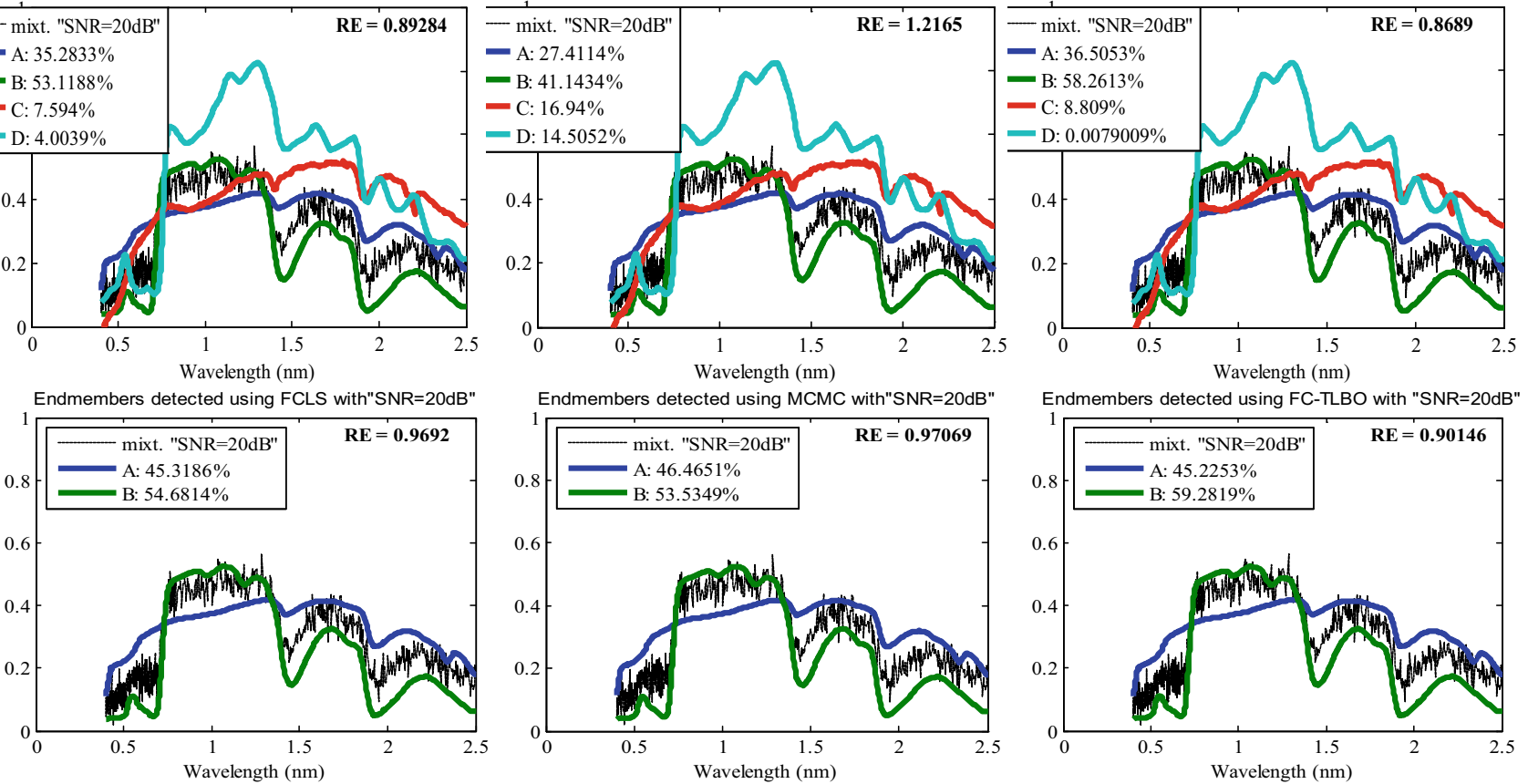

Figure 4. Performance of FC-TLBO with FCLS and MCMC with varying number of endmembers. First row represent comparisons in terms of unmixing confidence factor and reconstruction error between FC-TLBO and other two state-of-the-art algorithms for six endmember. The second and third rows represent the same comparison for four and two endmembers, respectively.

Zero-mean white Gaussian noise is added to the synthetic pixel to evaluate the robustness to noise with signal-tonoise ratio (SNR) defined as

$$
\mathrm{SNR}=10 \log _{10} \frac{E\left[y^{\prime} y\right]}{E\left[e^{\prime} e\right]}
$$


where $\|\bullet\|$ is normalisation operation and $E[\bullet]$ is the expectation maximisation, $M$ is a number of available pixels.

As stated before, the proposed algorithm and other two state-of-the-art algorithms were implemented using Matlab R2013a on a 64-bit Intel Core i5 processor with Windows 7 operating system.

\subsection{Dataset description}

4.1a Simulated scene: The simulated scene of pixels was synthesised using $K$ endmembers collected from USGS digital spectral library [20] as shown in figure 1. The simulated scene generation process is presented as follows.

- Randomly select $K$ endmembers from USGS spectral library to create endmember matrix $E$ of size $(K \times 224)$.

- Randomly create abundance Matrix $S$ of size $(3600 \times K)$ using dirichlet_rnd function.

- Multiply $S$ and $E$ to create matrix $Y$ of size $(3600 \times 224)$.

- Resize $Y$ to create a simulated scene of size $(60 \times 60 \times 224)$.

4.1b Cuprite dataset: The data are captured by NASA's AVIRIS sensor over the Cuprite mining district in Nevada [21]. The reflectances of some bands have been reduced due to water absorption and low SNR, leaving total 188 bands. Two regions of interest of size $(40 \times 40)$ were extracted for experimentation as shown in figure 6 .

4.1c Indian Pines dataset: Dataset and its ground truth are captured over the Indian Pines test site in Northwestern Indiana [22]. The scene consists of $145 \times 145$ pixels and 224 spectral bands in the wavelength range of $(0.4 \mathrm{~nm}-2.5 \mathrm{~nm})$. The reflectance of some bands [104-108]; [150-163] have been reduced due to water absorption and low SNR, leaving total 200 bands. This dataset is most widely used for hyperspectral data analysis. A false colour composite of datasets and its respective ground truth differentiation are shown in figure 10 .

4.1d The University of Pavia dataset: This scene and its ground truth were acquired by the ROSIS sensor over Pavia University, Northern Italy [22]. The Pavia University dataset consists of $610 \times 340$ pixels and 103 spectral bands. The geometric resolution is $1.3 \mathrm{~m}$ and ground truth differentiate the image in nine classes. A false colour composite of data is shown in figure 11 .

\subsection{Experiment 1 -simulated scene}

The experiments are carried out considering 14 endmembers from the USGS spectral library and randomly selected pixels from the simulated scene. The graphical representation of abundances estimated by FCLS, MCMC and FCTLBO algorithm is shown in figure 3. It is the notable thing that sometimes the estimated abundances using FCLS do not follow ANC. The abundances estimated by FC-TLBO achieve better accuracy compared with other two state-ofthe-art algorithms when the exact number of endmembers is known in advanced.

To verify the influence of noise on classification accuracy of the FC-TLBO algorithm, $20 \mathrm{~dB}$ white Gaussian noise is added to the original signal as shown in figure 4 . An experiment was performed by considering a different set of endmembers. The confidence factor and RE are considered evaluation parameters. It is observed from figure 4 that for a different set of endmembers, the endmember B has been selected as a choice by FCLS, MCMC and FC-TLBO. However, for the set of six endmembers the confidence factor of selection using FCLS, MCMC and FCTLBO is $53.12 \%, 37.38 \%$ and $59.44 \%$, respectively. Also, the RE using FCLS, MCMC and FC-TLBO is 0.89, 1.73 and 0.86 , respectively. The results for the set four and two endmembers also reveals that the confidence factor and RE using FC-TLBO outperforms the other two state-of-the-art algorithms.

Another experiment was performed to evaluate the performance of the proposed method under the influence of noise on the synthetic image. The synthetic image was created using eight endmembers selected from a USGS spectral library.

The zero-mean white Gaussian noise (from SNR $\infty$ to $15 \mathrm{~dB}$ ) is added to the randomly selected pixel from synthetic image. The REs for different values of SNR using FCLS, MCMC and FC-TLBO are shown in figure 5. FCTLBO shows better performance compared with other algorithms at different values of SNR. The practically evaluated RE values using FC-TLBO with varying SNR from $\infty$ to $15 \mathrm{~dB}$ shows its robustness to the added noise.

The synthetic image was synthesised using 14 endmembers from the USGS spectral library to evaluate the performance of proposed algorithm for a different combination of endmembers $(\mathrm{K})$. The REs for a different combination of endmembers using FCLS, MCMC and FCTLBO are given in figure 6. In particular, when an exact number (Real) of endmembers is known in advance, FCLS

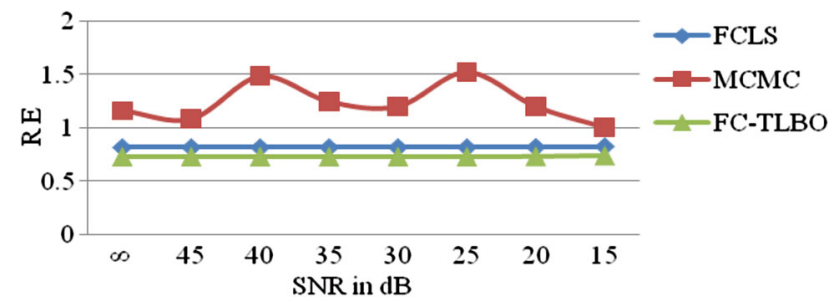

Figure 5. Comparative performance in terms of reconstruction error (RE) with varying SNR. 


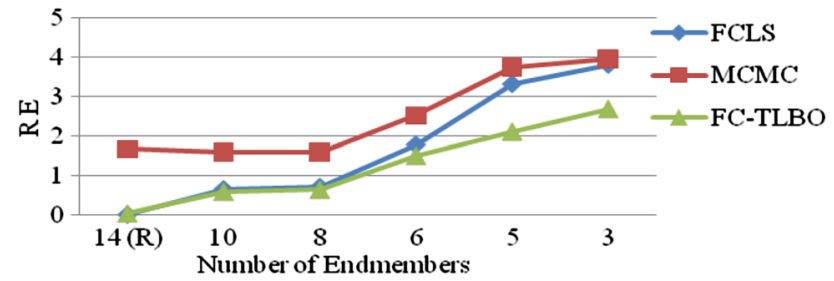

Figure 6. Reconstruction errors of simulated scene with different number of endmembers.

Table 1. Performance of FC-TLBO with different population size.

\begin{tabular}{lccccc}
\hline & $\mathrm{P}=5$ & $\mathrm{P}=20$ & $\mathrm{P}=30$ & $\mathrm{P}=40$ & $\mathrm{P}=50$ \\
\hline RMSE & 1.3096 & $2.70 \mathrm{E}-06$ & $1.80-04$ & $9.90 \mathrm{E}-04$ & $2.40 \mathrm{E}-07$ \\
RE & 0.3164 & 0.027 & 0.021 & 0.018 & 0.0162 \\
Time (s) & 271.14 & 675 & 981 & 1355 & 1747 \\
\hline
\end{tabular}

algorithm has better RE. However, the graph (in figure 6) shows that the FC-TLBO achieves better accuracy irrespective of knowledge of exact numbers of endmembers contributed in the spectral unmixing.

\subsection{Adjustable parameter analysis}

The population of random abundance fractions used in FCTLBO is an adjustable parameter, which may have a distinct effect on performance. The effect of different population size on unmixing errors and computing time is illustrated in table 1 . When the population size is more than five, the observed unmixing errors have small variations. The effect of population size on the computing time is also reported in table 1 . In this context, it is worthwhile to say that the population size of 20 is quite abreast of the unmixing errors like that of larger population size. However, we consider population size as 50 to achieve a more robust performance.

\subsection{Experiment 2-real hyperspectral scene}

The second experiment is accomplished using a scene captured by NASA's AVIRIS sensor over the Cuprite mining district in Nevada ${ }^{3}$. Reflectances of some bands have been reduced due to water absorption and low SNR, leaving total 188 bands. Two regions of interest of size $(40 \times 40)$ were extracted for experimentation as shown in figure 7. The RE of proposed FC-TLBO algorithm outperforms the other two state-of-the-art algorithms as shown in figure 8. As shown in figure 9, the endmembers selected for classification were extracted from the same image. The classification maps of the region of interest 1 (ROI 1) and region of interest 2 (ROI 2) using FCLS, MCMC and FCTLBO are also presented in figure 9. The color of a pixel

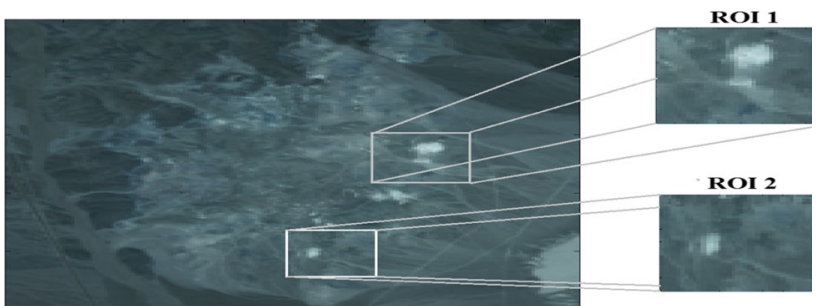

Figure 7. False colour composition of Cuprite image with bands [21 54 64] (left) and region of interest (right).

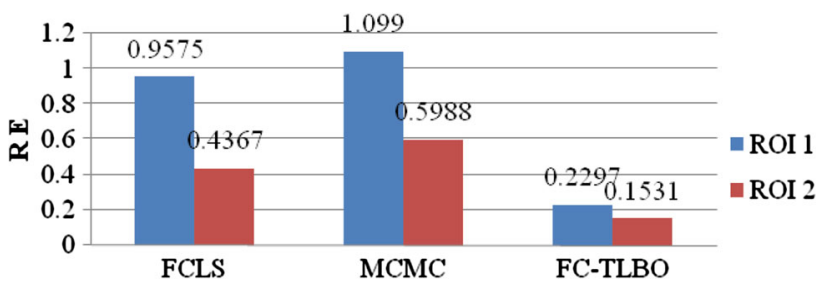

Figure 8. Reconstruction error of selected region of interest (ROI) from real Cuprite dataset.

represents the class of relative endmember. Since the ground truth of Cuprite dataset is not available, the classification accuracy of proposed FC-TLBO and other two state-of-the-art algorithms are evaluated using RE calculated by estimated abundance fractions and the set of selected endmembers.

The following steps are used to create the classification map of the respective dataset using MCMC, FCLS and FCTLBO algorithms.

- Calculate the abundance vector of all the pixels of a given image.

- For a given pixel, find the position of the greatest value of the respective abundance vector of size $(K \times 1)$.

- The position of the greatest value represents the classification of respective pixel to the particular endmember.

- Assign the colour of the respective endmember for the given pixel.

- Repeat the above three steps for each pixel of a given image.

- Display the classification map.

To evaluate the classification accuracy of the proposed FC-TLBO algorithm, two most widely used benchmark datasets (i.e., Indian Pines and University of Pavia) are used. The endmembers required for classification is extracted from the ground truth of respective hyperspectral images. The set of pixels of each class was retrieved by selecting 100 pixels from the respective ground truth in a random way. The endmembers used for classification are calculated by averaging the selected set of pixels in each class. For Indian Pines dataset, the three classes, that is, Alfalfa, Grass-pas-mowed and Oats, had less than 100 of 


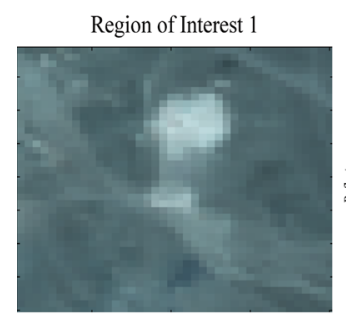

Region of Interest 2

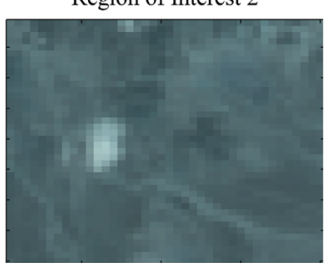

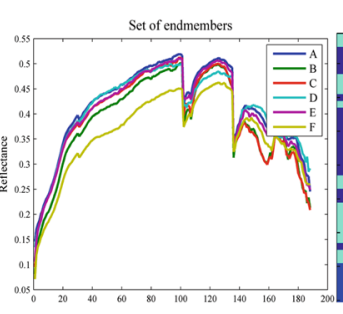

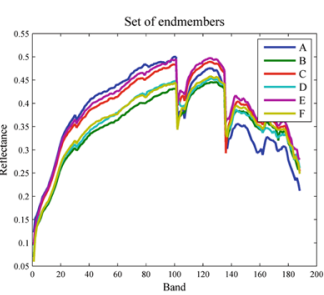

Classification by FCLS

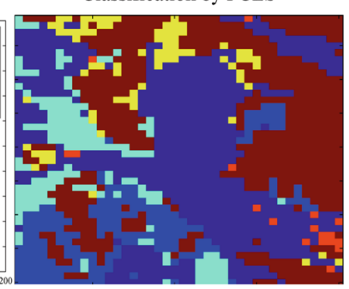

Classification by FCLS

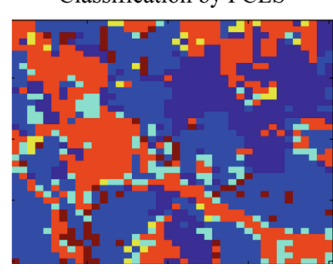

Classification by MCMC

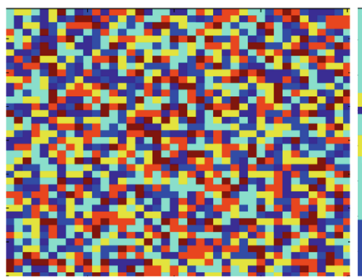

Classification by MCMC

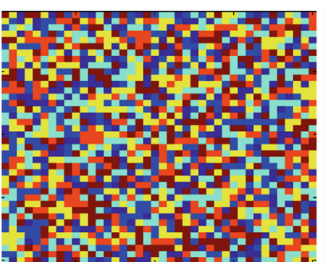

Classification by FC-TLBO

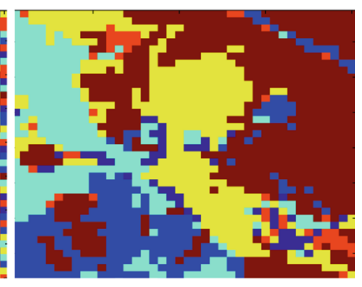

Classification by FC-TLBO

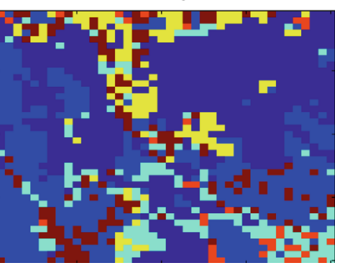

Figure 9. The classifications by FCLS, MCMC and FC-TLBO. The first and second rows contain an endmembers considered for unmixing and a pictorial representation of classification for regions of interest 1 and 2, respectively.

sample pixel. Therefore, for these classes all available samples were considered for endmembers creation. The graphical representation of endmembers selected for Indian Pines and University of Pavia dataset is available in figures 10 and 11, respectively. (Note: Since we are unable to find the fully constraints evolutionary approach of abundance estimation, the comparative analysis of the FCTLBO algorithm with other evolutionary algorithm is not included in the manuscript.)

To evaluate the performance of classification, overall accuracy (OA), average accuracy (AA) and Kappa coefficient are used as an evaluation parameter [23]. In detail, to evaluate the performance of classification accuracy, the statistical significance of the results has been computed. Since MCMC and FC-TLBO are based on evolutionary techniques, their time of execution can be different for different values of the initial parameter. Therefore, in all the aforementioned approaches, the time of execution is excluded in the comparative analysis. All the classification results have been obtained by using FCLS, MCMC and FC-TLBO algorithm depending on the values of abundance fractions. The dominant values among
False colour composition of Indian Pines dataset

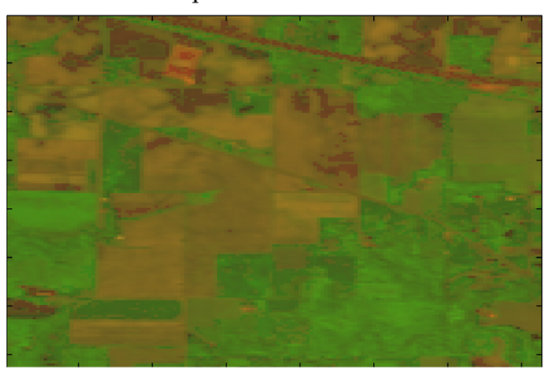

Classification using FCLS

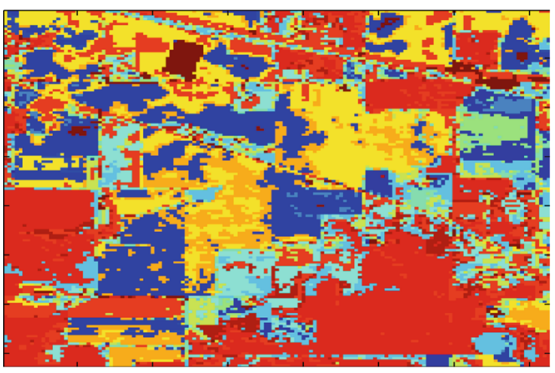

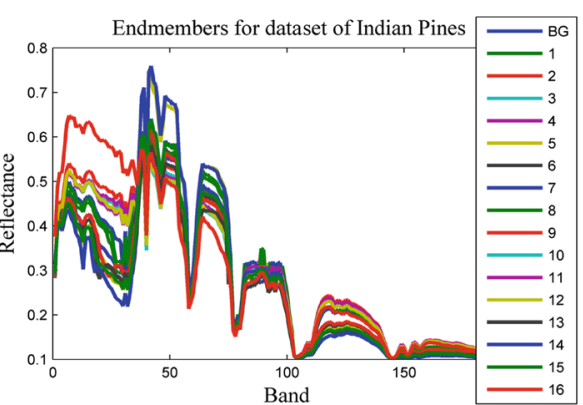

Classification using MCMC

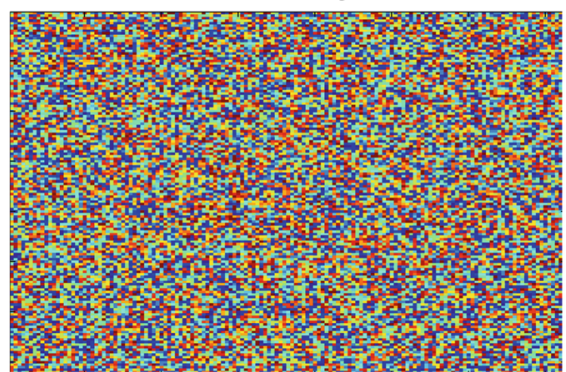

Ground Truth of Indian Pines

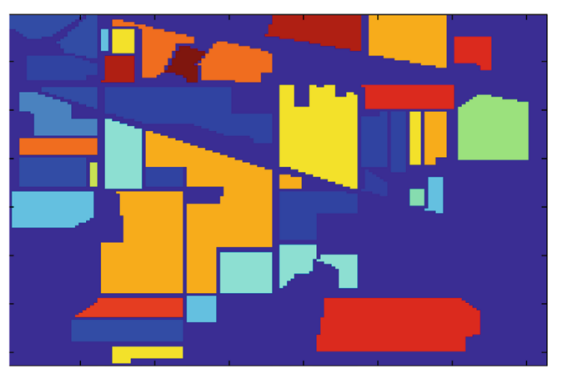

Classification using FC-TLBO

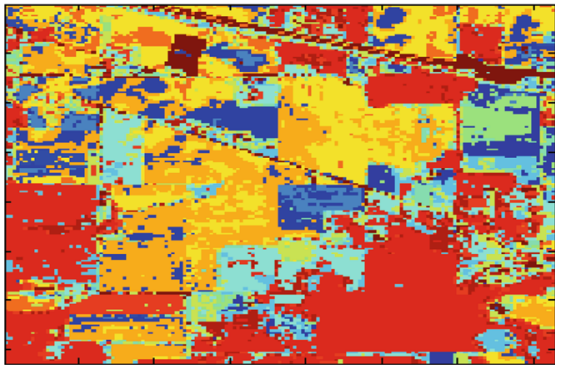

Figure 10. Performance of FC-TLBO with FCLS and MCMC for Indian Pines dataset. First row represents false colour composition of Indian Pines dataset, the endmember extracted from ground truth, and pictorial representation of ground truth, respectively. Second row contains the visual representation of classification map using FCLS, MCMC and FC-TLBO algorithms. 

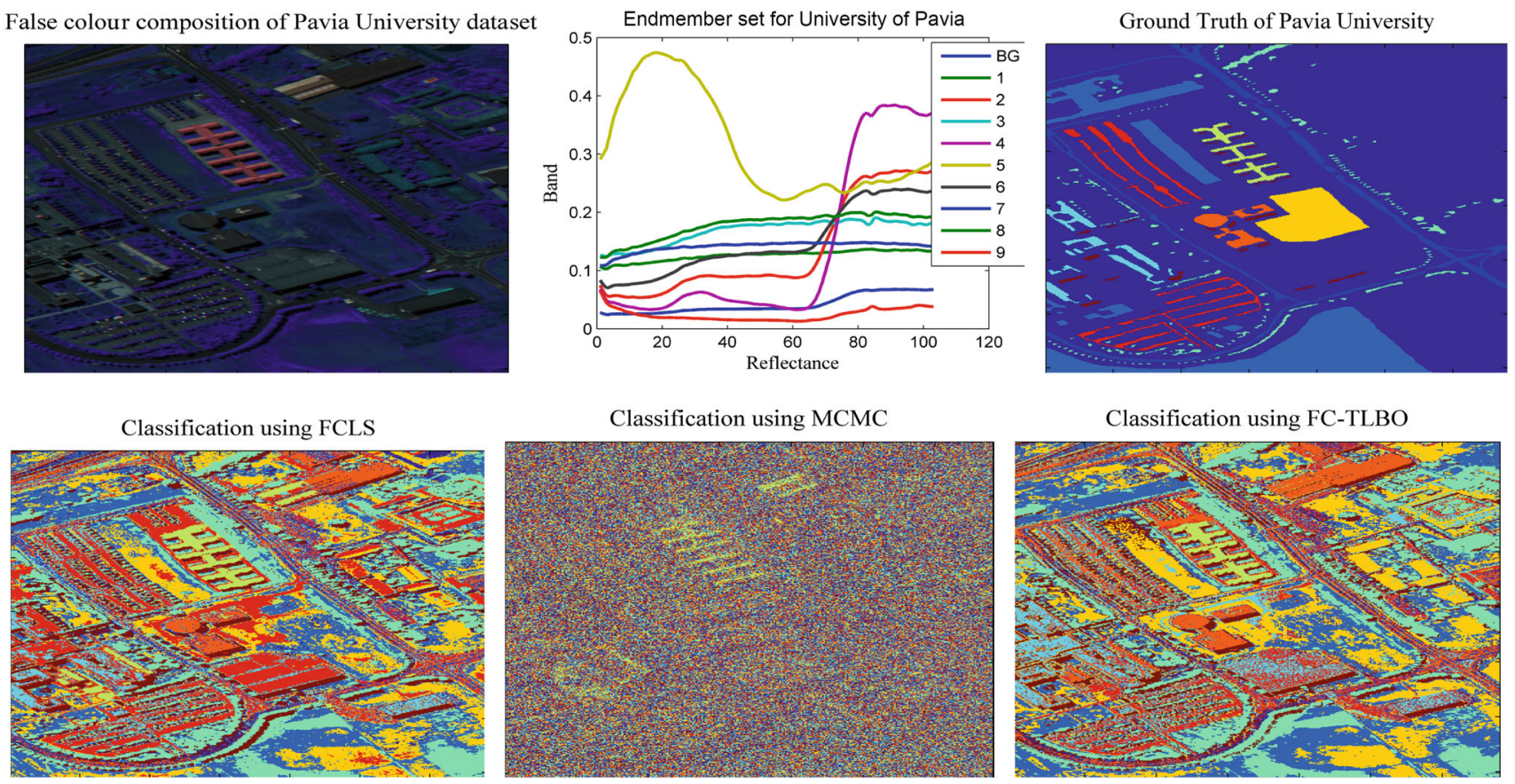

Figure 11. Performance of FC-TLBO with FCLS and MCMC for University of Pavia dataset. First row represents false colour composition of University of Pavia dataset, the endmember extracted from ground truth, and pictorial representation of ground truth, respectively. Second row contains the visual representation of classification map using FCLS, MCMC and FC-TLBO algorithms.

the abundances of the particular pixel are used to classify the pixel to the respective class.

The classification results obtained by using Indian Pines dataset are reported in table 2. Since the classifier used for an experiment depends on spectral values of the pixel, the pixels which are correctly classified according to the ground truth were only considered for the evaluation of classification. Looking at the final OA, AA and kappa

Table 2. Performance of FC-TLBO with FCLS and MCMC in terms of OA, AA and KAPPA for Indian Pines dataset.

\begin{tabular}{|c|c|c|c|c|c|c|c|c|}
\hline \multirow[b]{2}{*}{ Class } & \multirow[b]{2}{*}{ Class name } & \multirow[b]{2}{*}{ True samples } & \multicolumn{3}{|c|}{ Correctly estimated samples } & \multicolumn{3}{|c|}{ Class wise accuracy } \\
\hline & & & FCLS & MCMC & FC-TLBO & FCLS & MCMC & FC-TLBO \\
\hline Class 1 & Alfalfa & 46 & 44 & 3 & 43 & 95.652 & 6.5217 & 93.478 \\
\hline Class 2 & Corn-notill & 1428 & 769 & 83 & 478 & 53.852 & 5.8123 & 33.473 \\
\hline Class 3 & Corn-mintill & 830 & 104 & 47 & 153 & 12.53 & 5.6627 & 18.433 \\
\hline Class 4 & Corn & 237 & 28 & 15 & 83 & 11.814 & 6.3291 & 35.021 \\
\hline Class 5 & Grass-pasture & 483 & 22 & 24 & 34 & 4.554 & 4.9689 & 7.0393 \\
\hline Class 6 & Grass-trees & 730 & 395 & 47 & 526 & 54.11 & 6.4384 & 72.054 \\
\hline Class 7 & Grass-pas-mowed & 28 & 26 & 4 & 26 & 92.857 & 14.286 & 92.857 \\
\hline Class 8 & Hay-windrowed & 478 & 199 & 26 & 266 & 41.632 & 5.4393 & 55.648 \\
\hline Class 9 & Oats & 20 & 15 & 0 & 16 & 75 & 0 & 80 \\
\hline Class 10 & Soybean-notill & 972 & 489 & 64 & 510 & 50.309 & 6.5844 & 52.469 \\
\hline Class 11 & Soybean-mintill & 2455 & 741 & 167 & 1363 & 30.183 & 6.8024 & 55.519 \\
\hline Class 12 & Soybean-clean & 593 & 0 & 27 & 86 & 0 & 4.5531 & 14.502 \\
\hline Class 13 & Wheat & 205 & 199 & 10 & 194 & 97.073 & 4.878 & 94.634 \\
\hline Class 14 & Woods & 1265 & 1179 & 58 & 1230 & 93.202 & 4.585 & 97.233 \\
\hline Class 15 & Build-Gra-Tr-Driv & 386 & 26 & 21 & 46 & 6.735 & 5.4404 & 11.917 \\
\hline \multirow[t]{4}{*}{ Class 16} & Stone-Steel-Tow & 93 & 92 & 7 & 87 & 98.92 & 7.608 & 93.548 \\
\hline & & & & & OA & 42.23 & 5.8835 & 50.161 \\
\hline & & & & & AA & 51.15 & 5.9944 & 56.739 \\
\hline & & & & & KAPPA & 0.33 & 0.07 & 0.43 \\
\hline
\end{tabular}


Table 3. Performance of FC-TLBO with FCLS and MCMC in terms of OA, AA and KAPPA for University of Pavia dataset.

\begin{tabular}{|c|c|c|c|c|c|c|c|c|}
\hline \multirow[b]{2}{*}{ Class } & \multirow[b]{2}{*}{ Class name } & \multirow[b]{2}{*}{ True samples } & \multicolumn{3}{|c|}{ Correctly estimated samples } & \multicolumn{3}{|c|}{ Class-wise accuracy } \\
\hline & & & FCLS & MCMC & FC-TLBO & FCLS & MCMC & FC-TLBO \\
\hline Class 1 & Asphalt & 6631 & 2034 & 829 & 1521 & 30.674 & 12.5018 & 22.937 \\
\hline Class 2 & Meadows & 18649 & 8650 & 2204 & 9590 & 46.3831 & 11.8183 & 51.423 \\
\hline Class 3 & Gravel & 2099 & 1128 & 271 & 1622 & 53.7398 & 12.9109 & 77.274 \\
\hline Class 4 & Trees & 3064 & 3020 & 435 & 2978 & 98.5639 & 14.1971 & 97.193 \\
\hline Class 5 & Painted met. sheet & 1345 & 1335 & 651 & 1318 & 99.2565 & 48.4014 & 97.992 \\
\hline Class 6 & Bare soil & 5029 & 1547 & 575 & 2150 & 30.7615 & 11.4336 & 42.752 \\
\hline Class 7 & Bitumen & 1330 & 1166 & 169 & 1209 & 87.6691 & 12.7067 & 90.902 \\
\hline Class 8 & Self-Block. Bricks & 3682 & 2848 & 451 & 1749 & 77.3492 & 12.2487 & 47.501 \\
\hline \multirow[t]{4}{*}{ Class 9} & Shadows & 947 & 941 & 184 & 938 & 99.3664 & 19.4297 & 99.049 \\
\hline & & & & & OA & 52.9946 & 13.4865 & 53.943 \\
\hline & & & & & AA & 69.3071 & 17.2943 & 69.669 \\
\hline & & & & & KAPPA & 0.4513 & 0.062 & 0.4563 \\
\hline
\end{tabular}

coefficient values after application of the proposed FCTLBO and other algorithms, a significant improvement regarding classification accuracy has been achieved in most of the cases for Indian Pines dataset. As shown in figure 10, the OA values using FCLS, MCMC and FC-TLBO are $42.23 \%, 5.88 \%$ and $50.16 \%$, respectively, whereas the Kappa coefficients are $0.33,0.07$ and 0.43 , respectively.

The classification results obtained by using the University of Pavia dataset are reported in table 3. It is a notable thing that the slightly improved, but statistically similar OA and AA has been achieved by using an FCTLBO algorithm compared to FCLS algorithm. Whereas much better accuracy has been achieved by the FC-TLBO algorithm compared to MCMC algorithm. In the case of the FC-TLBO algorithm, slightly better OA, AA and Kappa coefficient $(53.94 \%, 69.67 \%$ and 0.4563$)$ has been achieved compared to OA, AA and Kappa coefficient $(52.99 \%$, $69.30 \%$ and 0.4513 ) using FCLS algorithm. This is because the endmembers used in classification significantly differ from each other, which increase the probability of correct endmember identification. In addition, according to figure 11, a visual interpretation of classification map using FC-TLBO shows an improvement in classification result compared to FCLS and MCMC method.

\section{Conclusion and prospective work}

In this paper, we have proposed an FC-TLBO algorithm for hyperspectral unmixing and its efficient implementation in a known and unknown environment. This algorithm is a representative method of a class of algorithms for spectral unmixing using meta-heuristic approach. Our experiments demonstrate that the FC-TLBO algorithm outperforms the other algorithms regarding better unmixing accuracy, especially when the contribution of exact endmembers in the hyperspectral scene is not known in advance. The experimental analysis also reveals that the proposed algorithm is robust to variation in signal-to-noise ratio. This approach can be extended to non-linear mixing models.

\section{Acknowledgement}

The authors would like to thank Dobigeon et al and Heinz et al for sharing their Matlab implementations of Markov chain Monte Carlo and fully constraints least square method, respectively. The authors would also like to thank Prof. Bhurchandi for his valuable suggestions, which contribute a lot to technical improvement of the manuscript.

\section{References}

[1] Goetz A, Vane G, Solomon J and Rock B 1985 Imaging spectrometry for earth remote sensing. Science 228: $1147-1153$

[2] Green R O et al 1998 Imaging spectroscopy and the airborne visible/infrared imaging spectrometer (AVIRIS). Remote Sens. Environ. 65(3): 227-248

[3] Hapke B W 1981 Bidirectional reflectance spectroscopy: 1. Theory. J. Geophys. Res. 86(B4): 3039

[4] Heylen R, Parente M and Gader P 2014 A review of nonlinear hyperspectral unmixing methods. IEEE J. Select. Topics Appl. Earth Observ. Remote Sens. 7(6): 1844-1865

[5] Dobigeon $\mathrm{N}$ et al 2013 Nonlinear unmixing of hyperspectral images. IEEE Signal Process. Mag. 31(1): 82-94. doi:10. 1109/MSP.2013.2279274

[6] Bing Zhang et al 2014 PSO-EM: a hyperspectral unmixing algorithm based on normal composition model. IEEE Trans. Geosci. Remote Sens. 52(12): 7782-7792

[7] Heinz D C et al 2001 Fully constrained least squares linear spectral mixing analysis method for material quantification in hyperspectral imagery. IEEE Trans. Geosci. Remote Sens. 39(3): 529-545 
[8] Yuan, Min Fu and Xiaoqiang Lu 2015 Substance dependence constrained sparse NMF for hyperspectral unmixing. IEEE Trans. Geosci. Remote Sens. 53(6): 2975-2986

[9] Dobigeon N et al 2013 MCMC algorithms for supervised and unsupervised linear unmixing of hyperspectral images. EAS Publ. Ser. 59(2013): 381-401. doi:10.1051/eas/1359017

[10] Ghamisi P and Benediktsson J A 2013 Feature selection based on hybridization of genetic algorithm and particle swarm optimization. IEEE Trans. Geosci. Remote Sens. 12(2): 309-313

[11] Zhong Y, Zhao L and Zhang L 2014 An adaptive differential evolution endmember extraction algorithm for hyperspectral remote sensing imagery. IEEE Geosci. Remote Sens. Lett. 11(6): 1061-1065

[12] Zhang B, Gao J, Gao L and Sun X 2013 Improvements in ant colony optimization algorithm for endmember extraction from hyperspectral images. IEEE J. Select. Topics Appl. Earth Observ. Remote Sens. 6(2): 522-530

[13] Pedergnana $M$ et al A novel technique for optimal feature selection in an attribute based on genetic algorithm. IEEE Trans. Geosci. Remote Sens. 51(6): 3514-3526

[14] Liu J and Zhang J 2014 Spectral unmixing via compressive sensing. IEEE Trans. Geosci. Remote Sens. 52(11): 099-7110

[15] Wang N, Du B, Zhang L and Zhang L 2015 An abundance characteristic based independent analysis for hyperspectral unmixing. IEEE Trans. Geosci. Remote Sens. 53(1): 416-428
[16] Broasdwater $\mathbf{J}$ and Banerjee A 2010 Nonlinear spectral mixture analysis for hyperspectral imagery in an unknown environment. IEEE Geosci. Remote Sens. Lett. 17(4): 836-840

[17] $\mathrm{Pu} \mathrm{H}$, Chen Z, Wang B and Xia W 2015 Constrained least square algorithm for nonlinear unmixing of hyperspectral imagery. IEEE Trans. Geosci. Remote Sens. 53(3): 1287-1302

[18] Rao R V and Pate V 2012 An elitist teaching-learning-based optimization algorithm for solving complex constrained optimization problems. Int. J. Ind. Eng. Comput. 3(4): $535-560$

[19] http://www.lx.it.pt/ bioucas/code.htm Visiting date: 10 May 2016

[20] http://speclab.cr.usgs.gov/spectral-lib.html Visiting date: 12 May 2016

[21] http://aviris.jpl.nasa.gov/data/free_data.html Visiting date: 1 June 2016

[22] http://www.ehu.eus/ccwintco/index.php?title=Hyperspec tral_Remote Visiting date: 1 June 2016

[23] Bakos K, Marpu P R and Gamba P 2011 Decision fusion of multiple classifiers for hyperspectral data classification. In: Optical remote sensing: advances in signal processing and exploitation techniques. In: Prasad S, Bruce L M and Chanussot J (eds.), 1st edn. New York: Springer-Verlag, ser. Augmented Vision and Reality Series 